\title{
PRIME ASSOCIATOR-DEPENDENT RINGS WITH IDEMPOTENT $\left({ }^{1}\right)$
}

BY

\author{
NICHOLAS J. STERLING
}

1. Introduction. Associator-dependent rings as a class were defined by Kleinfeld [2]. They are rings $R$ which satisfy the following identities.

$$
\alpha_{1}(x, y, z)+\alpha_{2}(y, z, x)+\alpha_{3}(z, x, y)+\alpha_{4}(x, z, y)+\alpha_{5}(z, y, x)+\alpha_{6}(y, x, z)=0
$$

for fixed $\alpha_{i}$ in some field of scalars and $x, y, z$ in $R$ where the associator is defined as $(x, y, z)=(x y) z-x(y z)$.

$$
(x, x, x)=0 .
$$

A linearization of (2) yields (1) with $\alpha_{i}=1$ for $i=1-6$. Since (2) alone is not strong enough to yield a structure theory for $R$ we assume that (2) does not imply (1).

This class of rings includes, among others, the right and left alternative rings, Lie-admissible rings, rings of type $(\gamma, \delta)$, flexible rings, and antiflexible rings.

Kleinfeld, Kosier, Osborn and Rodabaugh [3] showed that an associator-dependent ring $R$ must satisfy one of the following three identities.

$$
\begin{gathered}
\alpha(y, x, x)-(\alpha+1)(x, y, x)+(x, x, y)=0, \\
(y, x, x)=(x, y, x), \\
(x, y, z)+(y, z, x)+(z, x, y)=0 .
\end{gathered}
$$

We note that any ring satisfying (4) is anti-isomorphic to one satisfying (3) where $\alpha=0$. It is our purpose in this paper to show that if $R$ is a prime associatordependent ring satisfying (3) and having an idempotent $e \neq 0,1$ then when the characteristic of $R$ is prime to $6, R$ is alternative unless $\alpha=-1,1,-\frac{1}{2},-2$. It then follows immediately, from a result of Slater's [6], that $R$ can be embedded in a Cayley-Dickson algebra.

An example to show that this result is false when $\alpha=-1$ can be found in [4]. The residual cases where $\alpha=1,-\frac{1}{2},-2$ correspond, respectively, to flexible, left alternative, and right alternative rings. With the assumption of Lie-admissibility we have shown in a paper to be published elsewhere that if $R$ is a prime right or left alternative ring with an idempotent $e \neq 0,1$ and characteristic prime to 6 then

Received by the editors August 20, 1966.

$\left.{ }^{(}\right)$This paper was written under Contract 1520.1023, United States Army Research Office. It is a part of the author's doctoral dissertation written under the supervision of Professor Erwin Kleinfeld at Syracuse University. 
$R$ is associative and a prime, flexible, power-associative ring $R$ with an idempotent $e \neq 0,1$ and a Peirce decomposition relative to $e$ with the same restriction on characteristic is associative.

It is worth noting that an arbitrary primitive ring is prime [5]. Hence, by defining a suitable radical, the results of this paper could be extended to semisimple rings.

2. Preliminary results. Construction of ideals. Let $R$ be an associator-dependent ring satisfying (3) where $\alpha \neq-1,1,-\frac{1}{2},-2$. Furthermore, assume that $R$ has an idempotent $e \neq 0,1$. If the characteristic of $R$ is prime to 6 the following results hold in $R$ [3].

1. $R$ has the Peirce decomposition $R=R_{11}+R_{10}+R_{01}+R_{00}$ relative to $e$ where $x_{i j}$ belongs to $R_{i j}$ if and only if $e x=i x$ and $x e=j x$ where $i, j=0,1$. Moreover, the sum of the submodules $R_{i j}$ is direct.

2. The following relations hold for products of elements from $R$ when $\alpha \neq 0$.

$$
\begin{gathered}
R_{i j} R_{k p} \subseteq \delta_{j k} R_{i p} \text { where } i, j, k, p=0,1 \text { and } \delta_{j k} \text { is the Kronecker delta, except } \\
R_{i j}^{2} \subseteq R_{j i}, x_{i j}^{2}=0 \text { where } i=0,1 \text { and } j=1-i .
\end{gathered}
$$

When $\alpha=0$ all of the above relations hold except

$$
R_{i \imath} R_{i j} \subseteq R_{i j}+R_{j j}, R_{i j} R_{i i} \subseteq R_{j j} \text { and }\left(x_{i i} y_{i j}-y_{i j} x_{i i}\right) \text { is in } R_{i j} \text {. }
$$

3. When $\alpha=0, K=R_{10} R_{11}+R_{01} R_{00}$ is a trivial ideal of $R$.

4. The following relations exist for associators of $R$ when $\alpha \neq 0$. When $\alpha=0$, the same relations hold so long as $R$ has no trivial ideals. Let $\sigma$ be an arbitrary permutation of the arguments in an associator. Then when $i \neq j$,

$$
\begin{aligned}
& \left(\sigma x_{i i}, \sigma y_{i j}, \sigma z_{j i}\right)=0 \\
& \left(\sigma x_{i i}, \sigma y_{i j}, \sigma z_{i i}\right)=0 \\
& \left(\sigma x_{i i}, \sigma y_{j i}, \sigma z_{i i}\right)=0 \\
& \left(\sigma x_{i i}, \sigma y_{i j}, \sigma z_{j j}\right)=0 \\
& \left(\sigma x_{i i}, \sigma y_{i i}, \sigma z_{j j}\right)=0
\end{aligned}
$$

$(\sigma x, \sigma y, \sigma z)=\operatorname{sgn} \sigma(x, y, z)$ for $x, y, z$ in $R$ unless each of $x, y, z$ has a nonzero component in the same $R_{i i}$ submodule.

We now prove

LEMMA 1. Let $R$ satisf. (2) and (3) where $\alpha=0$. Then if $R$ is a prime ring elements from $R$ satisfy (6) and (8).

Proof. Since $R$ is prime it can contain no trivial ideals. Hence $K=0$. But then from (7), $x_{i i} y_{i j}$ belongs to $R_{i j}$ and (6) and (8) follow.

For the remainder of this paper we assume that $R$ is a prime ring. Clearly, from (8), $R$ is alternative if and only if $R_{11}$ and $R_{00}$ are alternative. We will prove that, in fact, these subrings must be associative. 
It is immediate from (8) that the following identities hold for all $x, y, z$ in $R$ except when $x, y, z$ all have nonzero components in the same $R_{i i}$ submodule.

$$
\begin{aligned}
(x y+y x) z & =x(y z)+y(x z), \\
z(x y+y x) & =(z x) y+(z y) x, \\
(z x) y+(y x) z & =z(x y)+y(x z) .
\end{aligned}
$$

The Teichmüller identity, true for an arbitrary ring $R$, will be of use to us. We state it below.

$$
0=F(x, y, z, w)=(x y, z, w)-(x, y z, w)+(x, y, z w)-x(y, z, w)-(x, y, z) w
$$

The next two lemmas have been proven for alternative rings [1]. We will show that they are still true under our more general hypotheses.

Lemma 2. If $B_{i}$ is an ideal of $R_{i i}$, then $D_{i}=B_{i}+R_{j i} B_{i}+B_{i} R_{i j}+\left(R_{j i} B_{i}\right) R_{i j}$ is an ideal of $R$, where $i=0,1$ and $j=1-i$.

Proof. First, $R_{i i} D_{i} \subseteq R_{i i} B_{i}+R_{i i}\left(B_{i} R_{i j}\right)$ from (6). However, $R_{i i} B_{i} \subseteq B_{i}$ and, from (8), $R_{i i}\left(B_{i} R_{i j}\right) \subseteq\left(R_{i i} B_{i}\right) R_{i j} \subseteq B_{i} R_{i j}$. Hence, $R_{i i} D_{i} \subseteq D_{i}$. Similarly, $D_{i} R_{i i} \subseteq D_{i}$.

Next, $R_{j i} D_{i} \subseteq R_{j i} B_{i}+R_{j i}\left(R_{j i} B_{i}\right)+R_{j i}\left(B_{i} R_{i j}\right)$ from (6). But, $R_{j i}\left(B_{i} R_{i j}\right) \subseteq\left(R_{j i} B_{i}\right) R_{i j}$ from (8). Also, from (9), $R_{j i}\left(R_{j i} B_{i}\right) \subseteq\left(R_{j i}^{2}\right) B_{i}+\left(B_{i} R_{j i}\right) R_{j i}+B_{i}\left(R_{j i}^{2}\right)$. Since $\left(R_{j i}^{2}\right) B_{i}$ $=\left(B_{i} R_{j i}\right) R_{j i}=0$, by $(6)$, we conclude that $R_{j i}\left(R_{j i} B_{i}\right) \subseteq B_{i}\left(R_{j i}^{2}\right) \subseteq B_{i} R_{i j}$. Thus, $R_{j i} D_{i} \subseteq D_{i}$. A similar argument shows that $D_{i} R_{i j} \subseteq D_{i}$.

Now, $R_{i j} D_{i} \subseteq R_{i j}\left(R_{j i} B_{i}\right)+R_{i j}\left(B_{i} R_{i j}\right)+R_{i j}\left[\left(R_{j i} B_{i}\right) R_{i j}\right]$ from (6). But, $R_{i j}\left(R_{j i} B_{i}\right)$ $\subseteq\left(R_{i j} R_{j i}\right) B_{i} \subseteq B_{i}$ from (8). Since $R_{i j}\left(B_{i} R_{i j}\right) \subseteq\left(R_{i j} B_{i}\right) R_{i j}+\left(R_{i j}^{2}\right) B_{i}+R_{i j}\left(R_{i j} B_{i}\right)$, from (9), and $\left(R_{i j} B_{i}\right) R_{i j}=R_{i j}\left(R_{i j} B_{i}\right)=0$, from (6), we have $R_{i j}\left(B_{i} R_{i j}\right) \subseteq\left(R_{i j}^{2}\right) B_{i} \subseteq R_{j i} B_{i}$. Finally, by (9), $R_{i j}\left[\left(R_{j i} B_{i}\right) R_{i j}\right] \subseteq\left[R_{i j}\left(R_{j i} B_{i}\right)\right] R_{i j}+\left(R_{i j}^{2}\right)\left(R_{j i} B_{i}\right)+R_{i j}\left[R_{i j}\left(R_{j i} B_{i}\right)\right]$. However, $R_{i j}\left[R_{i j}\left(R_{j i} B_{i}\right)\right]=0$ and $\left[R_{i j}\left(R_{j i} B_{i}\right)\right] R_{i j} \subseteq\left[\left(R_{i j} R_{j i}\right) B_{i}\right] R_{i j} \subseteq B_{i} R_{i j}$ from (6) and (8). Furthermore, $R_{i j}^{2}\left(R_{j i} B_{i}\right) \subseteq R_{j i}\left(R_{j i} B_{i}\right) \subseteq D_{i}$ from (6) and the fact that we have already shown that $R_{j i} D_{i} \subseteq D_{i}$. Hence, $R_{i j} D_{i} \subseteq D_{i}$. A similar argument shows that $D_{i} R_{j i} \subseteq D_{i}$.

Finally, we consider $R_{j j} D_{i} \subseteq R_{j j}\left(R_{j i} B_{i}\right)+R_{j j}\left[\left(R_{j i} B_{i}\right) R_{i j}\right]$ from (6). However, $R_{j j}\left(R_{j i} B_{i}\right) \subseteq\left(R_{j j} R_{j i}\right) B_{i} \subseteq D_{i}$ and $R_{j j}\left[\left(R_{j i} B_{i}\right) R_{i j}\right] \subseteq\left[\left(R_{j j} R_{j i}\right) B_{i}\right] R_{i j} \subseteq D_{i}$ from (8). Thus, $R_{j j} D_{i} \subseteq D_{i}$. Similarly, $D_{i} R_{j j} \subseteq D_{i}$.

We conclude that $R D_{i}$ and $D_{i} R$ are contained in $D_{i}$ and that $D_{i}$ is an ideal of $R$.

LEMMA 3. If $R_{10}^{2} R_{10}=R_{01}^{2} R_{01}=R_{10} R_{10}^{2}=R_{01} R_{01}^{2}=0$, then $H=R_{10}^{2}+R_{01}^{2}$ is an ideal of $R$.

Proof. First, consider $R_{i j}^{2} R \subseteq R_{i j}^{2} R_{i i}+R_{i j}^{2} R_{i j}+R_{i j}^{2} R_{j i}+R_{i j}^{2} R_{j j}$. Now, $R_{i j}^{2} R_{i j}=0$ by hypothesis. Also, $R_{i j}^{2} R_{j j}=0$ and $R_{i j}^{2} R_{j i} \subseteq R_{j i}^{2}$ from (6). Since

$$
R_{i j}^{2} R_{i i} \subseteq R_{i j}\left(R_{i j} R_{i i}\right)+R_{i j}\left(R_{i i} R_{i j}\right)+\left(R_{i j} R_{i i}\right) R_{i j}
$$

from (9), and $R_{i j}\left(R_{i j} R_{i i}\right)=\left(R_{i j} R_{i i}\right) R_{i j}=0$ by (8), we conclude that $R_{i j}^{2} R_{i i} \subseteq R_{i j}^{2}$. Therefore $H R \subseteq H$. A similar argument yields $R H \subseteq H$. Thus $H$ is an ideal of $R$. 
We now proceed with other lemmas preliminary to our main result.

LemmA 4. $L=R_{i j} R_{j i}$ is an ideal in $R_{i i}$ in the nucleus of $R_{i i}$ where $i=0,1$ and $j=1-i$.

Proof. It is clear from (6) and (8) that $L$ is an ideal of $R_{i i}$. We will show that $L$ is in the left nucleus of $R$ and note that a similar argument places $L$ in the middle and right nuclei of $R$.

Let $x_{i j} \in R_{i j}, y_{j i} \in R_{j i}$ and $z_{i i}, w_{i i} \in R_{i i}$. Then

$$
\left(x_{i j} y_{j i}, z_{i i}, w_{i i}\right)=\left[\left(x_{i j} y_{j i}\right) z_{i i}\right] w_{i i}-\left(x_{i j} y_{j i}\right)\left(z_{i i} w_{i i}\right)=0
$$

by repeated applications of (8). Hence, $L$ is in the left nucleus of $R_{i i}$.

LeMMA 5. $R_{j i}^{2} R_{j i}$ and $R_{i j} R_{i j}^{2}$ are ideals in the center of $R_{i i}$ when $i=0,1$ and $j=1-i$.

Proof. It is immediate from Lemma 4 that $R_{j i}^{2} R_{j i}$ and $R_{i j} R_{i j}^{2}$ are in the nucleus of $R_{i i}$. Also, $\left(R_{j i}^{2} R_{j i}\right) R_{i i} \subseteq R_{j i}^{2}\left(R_{j i} R_{i i}\right) \subseteq R_{j i}^{2} R_{j i}$ and $R_{i i}\left(R_{i j} R_{i j}^{2}\right) \subseteq\left(R_{i i} R_{i j}\right) R_{i j}^{2} \subseteq R_{i j} R_{i j}^{2}$ from (6) and (8). Thus, $R_{j i}^{2} R_{j i}$ is a right ideal and $R_{i j} R_{i j}^{2}$ a left ideal, respectively, of $R_{i i}$. Therefore, to prove that they are both ideals in the center of $R_{i i}$, it suffices to show that they commute with each element in $R_{i i}$.

Before proceeding, we recall from (6) that $x_{i j}^{2}=0$ for $i \neq j$. Replacing $x_{i j}$ by $x_{i j}+y_{i j}$ we obtain

$$
x_{i j} y_{i j}+y_{i j} x_{i j}=0 \text {. }
$$

Now, let $x_{j i}, y_{j i}, z_{j i}$ belong to $R_{j i}$ and $w_{i i}$ to $R_{i i}$. Then

$$
\left[\left(x_{j i} y_{j i}\right) z_{j i}\right] w_{i i}=\left(x_{j i} y_{j i}\right)\left(z_{j i} w_{i i}\right)
$$

from (8). But,

$$
\left(x_{j i} y_{j i}\right)\left(z_{j i} w_{i i}\right)+\left[x_{j i}\left(z_{j i} w_{i i}\right)\right] y_{j i}=x_{j} \cdot\left[y_{j i}\left(z_{j i} w_{i i}\right)\right]+x_{j i}\left[\left(z_{j i} w_{i i}\right) y_{j i}\right]=0
$$

from (9) and (11). Hence,

$$
\left(x_{j i} y_{j i}\right)\left(z_{j i} w_{i i}\right)=-\left[x_{j i}\left(z_{j i} w_{i i}\right)\right] y_{j i}=\left(x_{j i}, z_{j i}, w_{i i}\right) y_{j i}=-\left(w_{i i}, z_{j i}, x_{j i}\right) y_{j i}
$$

from (6) and (8), and so,

$$
\left[\left(x_{j i} y_{j i}\right) z_{j i}\right] w_{i i}=-\left(w_{i i}, z_{j i}, x_{j i}\right) y_{j i} .
$$

However, from (10), we have

$$
\begin{aligned}
0=F\left(w_{i i}, z_{j i}, x_{j i}, y_{j i}\right)=\left(w_{i i} z_{j i},\right. & \left.x_{j i}, y_{j i}\right)-\left(w_{i i}, z_{j i} x_{j i}, y_{j i}\right) \\
& +\left(w_{i i}, z_{j i}, x_{j i} y_{j i}\right)-w_{i i}\left(z_{j i}, x_{j i}, y_{j i}\right)-\left(w_{i i}, z_{j i}, x_{j i}\right) y_{j i} .
\end{aligned}
$$

Whence, from (6) and (8), we get

$$
w_{i i}\left(z_{j i}, x_{j i}, y_{j i}\right)=-\left(w_{i i}, z_{j i}, x_{j i}\right) y_{j i} \text {. }
$$

Combining (12) and (13), we obtain,

$$
\left[\left(x_{j i} y_{j i}\right) z_{j i}\right] w_{i i}=w_{i i}\left(z_{j i}, x_{j i}, y_{j i}\right)=w_{i i}\left(x_{j i}, y_{j i}, z_{j i}\right)=w_{i i}\left[\left(x_{j i} y_{j i}\right) z_{j i}\right]
$$


from (6) and (8). We conclude that elements from $R_{j i}^{2} R_{j i}$ commute with elements from $R_{i i}$. A similar proof shows that elements from $R_{i j} R_{i j}^{2}$ also commute with elements from $R_{i i}$.

The next two lemmas deal with arbitrary prime rings.

Lemma 6. Let $R$ be a nonassociative prime ring. Then $R$ can contain no nuclear ideals.

Proof. Let $B$ be an ideal in the nucleus of $R$. Let $b$ belong to $B$ and $x, y, z$ to $R$. From (10) we obtain

$$
\begin{aligned}
0=F(b, x, y, z) & =(b x, y, z)-(b, x y, z)+(b, x, y z)-b(x, y, z)-(b, x, y) z \\
& =-b(x, y, z) .
\end{aligned}
$$

Further, $b[(x, y, z) w]=[b(x, y, z)] w=0$ where $w$ belongs to $R$. Hence,

$$
B(R, R, R)=B[(R, R, R) R]=0 .
$$

However, finite sums of elements of the form $(R, R, R)$ and $(R, R, R) R$ form an ideal in an arbitrary ring. Since $R$ is prime, $B$ must be zero unless $R$ is associative.

LEMMA 7. Let $R$ be an arbitrary prime ring. Then the set of annihilators of a nonzero element in the center of $R$ is zero.

Proof. Let $c \neq 0$ belong to the center of $R$ and suppose $x$ belongs to $R$ and $x c=c x=0$. Then $c(x R)=(x c) R=0$ and $c(R x)=(R x) c=R(x c)=0$. Therefore the annihilator of $c$ is an ideal of $R$, call it $B$. It is clear that $B$ annihilates the ideal generated by $c$. Sin : $c$ is assumed nonzero, we conclude that $B=0$.

3. Main section. The next lemma is crucial to the proof of our main result.

Lemma 8. Let $C_{i}$ and $B_{i}$ be ideals in $R_{i i}$ such that $B_{i} C_{i}=C_{i} B_{i}=0$. Then

and

$$
D_{i}=B_{i}+R_{j i} B_{i}+B_{i} R_{i j}+\left(R_{j i} B_{i}\right) R_{i j}
$$

$$
F_{i}=C_{i}+R_{j i} C_{i}+C_{i} R_{i j}+\left(R_{j i} C_{i}\right) R_{i j}
$$

are ideals of $R$ and $D_{i} F_{i}=F_{i} D_{i}=0$.

Proof. From Lemma 4, $F_{i}$ and $D_{i}$ are ideals of $R$. We have left to show that they annihilate one another. First, from (6) we have $D_{i} C_{i} \subseteq B_{i} C_{i}+\left(R_{j} B_{i}\right) C_{i}$. However, $B_{i} C_{i}=0$ by hypothesis and $\left(R_{j i} B_{i}\right) C_{i} \subseteq R_{j i}\left(B_{i} C_{i}\right)=0$ from (8). A similar argument applies to $C_{i} D_{i}, F_{i} B_{i}$, and $B_{i} F_{i}$ and we can conclude that

$$
D_{i} C_{i}=C_{i} D_{i}=F_{i} B_{i}=B_{i} F_{i}=0 .
$$

Next, $\quad D_{i}\left(R_{j i} C_{i}\right) \subseteq\left(R_{j i} B_{i}\right)\left(R_{j i} C_{i}\right)+\left(B_{i} R_{i j}\right)\left(R_{j i} C_{i}\right)+\left[\left(R_{j i} B_{i}\right) R_{i}\right]\left(R_{j i} C_{i}\right)$ from (6). From (9), $\left(R_{j i} B_{i}\right)\left(R_{j i} C_{i}\right) \subseteq R_{j i}\left[B_{i}\left(R_{j i} C_{i}\right)\right]+B_{i}\left[R_{j i}\left(R_{j i} C_{i}\right)\right]+\left(B_{i} R_{j i}\right)\left(R_{j i} C_{i}\right)$. However, $\left(B_{i} R_{j i}\right)\left(R_{j i} C_{i}\right)=R_{j i}\left[B_{i}\left(R_{j i} C_{i}\right)\right]=0$ from (6) and $B_{i}\left[R_{j i}\left(R_{j i} C_{i}\right)\right]=0$ from (14). Hence, $\left(R_{j i} B_{i}\right)\left(R_{j i} C_{i}\right)=0$. Finally, $\left(B_{i} R_{i j}\right)\left(R_{j i} C_{i}\right) \subseteq B_{i}\left[R_{i j}\left(R_{j i} C_{i}\right)\right]=0$ and $\left[\left(R_{j i} B_{i}\right) R_{i j}\right]\left(R_{j i} C_{i}\right)$ 
$\subseteq\left\{\left[\left(R_{j i} B_{i}\right) R_{i j}\right] R_{j i}\right\} C_{i}=0$ from (8) and (14). A similar argument applies to $F_{i}\left(R_{j i} B_{i}\right)$ and we have,

$$
D_{i}\left(R_{j i} C_{i}\right)=F_{i}\left(R_{j i} B_{i}\right)=0 .
$$

Now, $\left(R_{j i} C_{i}\right) D_{i} \subseteq\left(R_{j i} C_{i}\right)\left(B_{i} R_{i j}\right)$ from (6), (14) and (15). But,

$$
\left(R_{j i} C_{i}\right)\left(B_{i} R_{i j}\right) \subseteq R_{j i}\left[C_{i}\left(B_{i} R_{i j}\right)\right]=0
$$

from (8) and (15). Hence, as a similar argument applies to $\left(R_{j i} B_{i}\right) F_{i}$, we obtain

$$
\left(R_{j i} C_{i}\right) D_{i}=\left(R_{j i} B_{i}\right) F_{i}=0 .
$$

Next, $D_{i}\left(C_{i} R_{i j}\right) \subseteq\left(B_{i} R_{i j}\right)\left(C_{i} R_{i j}\right)$ from (6), (14) and (15). However,

$$
\left(B_{i} R_{i j}\right)\left(C_{i} R_{i j}\right) \subseteq B_{i}\left[R_{i j}\left(C_{i} R_{i j}\right)\right]+R_{i j}\left[B_{i}\left(C_{i} R_{i j}\right)\right]+\left(R_{i j} B_{i}\right)\left(C_{i} R_{i j}\right)
$$

from (9). Since $\left(R_{i j} B_{i}\right)\left(C_{i} R_{i j}\right)=0$ from (6) and $B_{i}\left[R_{i j}\left(C_{i} R_{i j}\right)\right]=R_{i j}\left[B_{i}\left(C_{i} R_{i j}\right)\right]=0$ from (14), we conclude that $\left(B_{i} R_{i j}\right)\left(C_{i} R_{i j}\right)=0$. A similar argument applies to $F_{i}\left(B_{i} R_{i j}\right)$. Hence

$$
D_{i}\left(C_{i} R_{i j}\right)=F_{i}\left(B_{i} R_{i j}\right)=0 .
$$

We have $\left(C_{i} R_{i j}\right) D_{i} \subseteq\left(C_{i} R_{i j}\right)\left[\left(R_{j i} B_{i}\right) R_{i j}\right]$ from (14), (15) and (17). However,

$$
\left(C_{i} R_{i j}\right)\left[\left(R_{j i} B_{i}\right) R_{i j}\right] \subseteq C_{i}\left\{R_{i j}\left[\left(R_{j i} B_{i}\right) R_{i j}\right]\right\}=0
$$

from (8) and (14). A similar argument applies for $\left(B_{i} R_{i j}\right) F_{i}$ and we obtain

$$
\left(C_{i} R_{i j}\right) D_{i}=\left(B_{i} R_{i j}\right) F_{i}=0 .
$$

Finally, we consider $\left[\left(R_{j i} C_{i}\right) R_{i j}\right] D_{i}$ and $D_{i}\left[\left(R_{j i} C_{i}\right) R_{i j}\right]$. From (14)-(18) it is clear that we need only consider $\left[\left(R_{j i} C_{i}\right) R_{i j}\right]\left[\left(R_{j i} B_{i}\right) R_{i j}\right]$. But,

$$
\left[\left(R_{j i} C_{i}\right) R_{i j}\right]\left[\left(R_{j i} B_{i}\right) R_{i j}\right] \subseteq\left(R_{j i} C_{i}\right)\left\{R_{i j}\left[\left(R_{j i} B_{i}\right) R_{i j}\right]\right\}=0
$$

from (8) and (17). Hence,

Similarly,

$$
\left[\left(R_{j i} C_{i}\right) R_{i j}\right] D_{i}=D_{i}\left[\left(R_{j i} C_{i}\right) R_{i j}\right]=0
$$

$$
\left[\left(R_{j i} B_{i}\right) R_{i j}\right] F_{i}=F_{i}\left[\left(R_{j i} B_{i}\right) R_{i j}\right]=0 .
$$

We conclude that $D_{i} F_{i}=F_{i} D_{i}=0$.

COROLlaRY. If $R$ is a prime ring then the subrings $R_{i i}$, where $i=0,1$ are also prime rings.

Proof. Let $C_{i}$ and $B_{i}$ be ideals of $R_{i i}$ such that $C_{i} B_{i}=B_{i} C_{i}=0$. Then $D_{i}$ and $F_{i}$, constructed as in Lemma 2, are ideals of $R$ such that $D_{i} F_{i}=F_{i} D_{i}=0$. Since $R$ is prime we conclude that either $D_{i}$ or $F_{i}$ is zero. Hence, either $B_{i}$ or $C_{i}$ is zero and $R_{i i}$ is a prime ring.

We now proceed to analyze the $R_{i i}$ spaces. Our attention is directed toward the four subspaces $R_{i j} R_{i j}^{2}$ and $R_{j i}^{2} R_{j i}$ where $i=0,1$ and $j=1-i$. 
First, suppose $R_{10}^{2} R_{10}=R_{01}^{2} R_{01}=R_{10} R_{10}^{2}=R_{01} R_{01}^{2}=0$. Then from Lemma 3 $H=R_{10}^{2}+R_{01}^{2}$ is an ideal of $R$.

Lemma 9. Let $R_{i j} R_{i j}^{2}=R_{j i}^{2} R_{j i}=0$ where $i=0,1$ and $j=1-i$. Then $H=R_{10}^{2}+R_{01}^{2}=0$.

Proof. We have $R_{i j}^{2} R_{j i}^{2}=0$ for $i \neq j$ from (6) and the hypothesis. Also,

$$
R_{i j}^{2} R_{i j}^{2} \subseteq R_{i j}\left(R_{i j} R_{i j}^{2}\right)+R_{i j}\left(R_{i j}^{2} R_{i j}\right)+\left(R_{i j} R_{i j}^{2}\right) R_{i j}
$$

from (9). Since all elements on the right of this inclusion relation vanish, $R_{i j}^{2} R_{i j}^{2}=0$. Thus, $H$ is a trivial ideal of $R$. Since $R$ is a prime ring, it follows that $H=0$.

LemMA 10. Let $H=R_{10}^{2}+R_{01}^{2}=0$. Then $B=R_{10} R_{01}+R_{10}+R_{01}+R_{01} R_{10}$ is an ideal in the nucleus of $R$.

Proof. From (6) and (8) it is evident that $B$ is an ideal of $R$.

Recall, from (8), that all associators containing at least one argument from $R_{i j}$, where $i \neq j$, alternate. Thus, to prove that $R_{i j}$ is in the nucleus of $R$, it suffices to show that $R_{i j}$ is in the left nucleus of $R$.

To this end, consider associators of the form $\left(R_{i j}, R_{k p}, R_{m n}\right)$ where $k, p, m, n$ $=0,1$. From (8), it is evident that unless two of the arguments are from the $R_{i j}$ or the $R_{j i}$ space the associator vanishes. But, $\left(R_{i j}, R_{i j}, R_{m n}\right)=\left(R_{i j}, R_{j i}, R_{j i}\right)=0$ regardless of the value $m$ assumes, from (6) and the fact that $H=0$. Hence, $R_{i j}$ is in the nucleus of $R$.

From Lemma 4, $R_{i j} R_{j i}$, where $i=0,1$ and $j=1-i$, is in the nucleus of $R_{i i}$. Since $R_{i j}, R_{j i}$ are in the nucleus of $R$ and associators of the form $\left(\sigma R_{i i}, \sigma R_{j j}, \sigma R_{j j}\right)=0$ from (8), where $\sigma$ is an arbitrary permutation of the three arguments, it follows that $R_{i j} R_{j i}$ is in the nucleus of $R$.

COROllary. $B=0$ if $R$ is not associative.

Proof. Immediate from Lemmas 6 and 10 .

LeMmA 11. If $R_{i j}^{2} R_{i j}=R_{j i} R_{j i}^{2}=0$ for $i=0,1$ and $j=1-i$ then $R$ is associative.

Proof. From Lemma 10, Corollary, we obtain $R=R_{11}+R_{00}$. Since $R_{11}$ and $R_{00}$ are orthogonal subrings of $R$ we conclude that they are, in fact, orthogonal ideals of $R$. Since $e \neq 0$ belongs to $R_{11}$, we get $R_{00}=0$. But then $R=R_{11}$ and $e$ becomes the identity for $R$, contrary to hypothesis. Hence, $R$ must be associative.

It follows that, unless $R$ is associative, at least one of the subspaces $R_{10}^{2} R_{10}$, $R_{01}^{2} R_{01}, R_{10} R_{10}^{2}, R_{01} R_{01}^{2}$ must not vanish. We will show that, under this condition, both $R_{11}$ and $R_{00}$ must be associative and $R$ must be alternative.

Assume, without loss of generality, that $R_{01} R_{01}^{2} \neq 0$. From Lemma $5, R_{01} R_{01}^{2}$ is a nonzero ideal in the center of $R_{00}$. Hence, from Lemma $6, R_{00}$ must be associative.

Recall, from Lemma 4, that $R_{10} R_{01}$ is an ideal in the nucleus of $R_{11}$. Unless $R_{11}$ is also associative, $R_{10} R_{01}=0$. Under the assumption that $R_{10} R_{01}=0$, we examine elements of the form $x_{01} y_{10}$ belonging to $R_{01} R_{10}$. From (8), we have

$$
\left(x_{01} y_{10}\right)^{2}=\left[\left(x_{01} y_{10}\right) x_{01}\right] y_{10}, \text { and }\left(x_{01}, y_{10}, x_{01}\right)=0 \text {. }
$$


Expanding this associator, we obtain $\left(x_{01} y_{10}\right) x_{01}-x_{01}\left(y_{10} x_{01}\right)$. However, $x_{01}\left(y_{10} x_{01}\right)=0$, and we obtain

$$
\left(x_{01} y_{10}\right) x_{01}=0=\left(x_{01} y_{10}\right)^{2} .
$$

In particular, $\left[x_{01}\left(y_{01} z_{01}\right)\right]^{2}=0$ for $x_{01}, y_{01}, z_{01}$ in $R_{01}$. From Lemmas 5 and 7 , it follows that $x_{01}\left(y_{01} z_{01}\right)=0$ and so, $R_{01} R_{01}^{2}=0$. This is contrary to our assumption. The contradiction arises from the assumption that $R_{10} R_{01}=0$. Thus, $R_{10} R_{01} \neq 0$ and, hence, $R_{11}$ must be associative.

We have proven

THEOREM. Let $R$ be a prime associator-dependent ring satisfying (3) where $\alpha \neq-1,1,-\frac{1}{2},-2$ and the characteristic of $R$ is prime to 6 . If $R$ has an idempotent $e \neq 0,1$ then $R$ is alternative.

\section{BIBLIOGRAPHY}

1. A. A. Albert, On simple alternative rings, Canad. J. Math. 4 (1952), 129-135.

2. E. Kleinfeld, Associator-dependent rings, Arch. Math. 13 (1962), 203-212.

3. E. Kleinfeld, F. Kosier, J. M. Osborn and D. Rodabaugh, The structure of associatordependent rings, Trans. Amer. Math. Soc. 110 (1964), 473-483.

4. F. Kosier, On a class of non-associative algebras, Trans. Amer. Math. Soc. 102 (1962), 299-318.

5. R. L. San Soucie, Weakly standard rings, Amer. J. Math. 79 (1957), 80-86.

6. M. B. Slater, Weakly prime alternative rings, Abstract 65T-166, Notices Amer. Math. Soc. 12 (1965), 367-368.

SYRACUSE UNIVERSITY, SYRACUSE, NEW YORK

State University of New York, Binghampton, New YoRK 